\title{
Chaotic Sensors and Tags: Design and Performances
}

\author{
Horia-Nicolai Teodorescu ${ }^{1,2}$ \\ 1 'Gh. Asachi' Technical University of Iasi \\ ${ }^{2}$ Institute of Computer Science of the Romanian Academy \\ Iasi, Romania \\ hteodor@etti.tuiasi.ro
}

\begin{abstract}
Chaotic tags and sensors can be conveniently made using simple chaotic circuits of reduced complexity that can compete with tags and sensors based on a combination of capacitance-, inductance-, or resistance-to-voltage converters and additional microcontrollers. The design of such devices is exemplified and details of the design discussed.
\end{abstract}

Keywords- chaotic sensor, nonlinear dynamics, chaotic electronic circuit, multimodal oscillation, $R F$ tags chaotic circuits, level sensor, level tags

\section{INTRODUCTION}

Value-to-frequency converters are commonly used for sensing, where 'value' may represent capacitance [1], [2], inductance [3], or resistance. A direct generalization of these devices consists of chaotic oscillators with the nonlinear dynamics (operation) dependent on the value of a sensing element, with the sensing part being an ambient-dependent capacitor, inductor, or resistor. Nonlinear dynamic systems in sensing have been introduced in relation with various applications including distance measurement [4], [5], conductivity and conductive titrimetry [6], frequency discrimination [7], blood glucose noninvasive measurement [8], artificial retinas and basic image pattern recognition [9], metal detectors [10,11], and dc voltage detectors [12]. Sensors able to transmit data using the signal generated by the nonlinear dynamics were reported in [13].

We discuss sensors where the sensing element can be capacitive, resistive, or inductive and can be easily made in a thin configuration suitable for tag and for wearable sensors. A single chaotic sensing circuit is presented that can fit a large range of applications; the circuit generalizes and builds on the scheme introduced in $[6,14]$. The presented design of the sensors can be relatively easy trimmed to operate as a RF tag fitting an available frequency $(124,125$, and $135 \mathrm{kHz}$, or $13.56 \mathrm{MHz}$ [15]).

In Section II, the principles of chaotic sensors are introduced; in Section III we present the design principles and an example; Section IV addresses the issue of information extraction from the chaotic signal; applications are discussed in Section $\mathrm{V}$, while in Section VI we discuss the results and conclude the paper.

\section{PRINCIPLES AND LIMITS OF THE CHAOTIC SENSORS}

Chaotic sensors are essentially parameter (resistance, capacitance, or inductance) to voltage converters of a specific type, based on parametercontrolled variable frequency and variable waveform oscillators. Chaotic circuits for chaotic sensors behave for some values of the measured variable as periodic oscillators. The difference between the chaotic sensors and common oscillating sensors is that the former take advantage of the chaotic behavior to boost their sensitivity and multi-parameter sensing capability.

Chaotic systems in general and chaotic sensors as a particular case of chaotic systems are driven by differential equations that are nonlinear and of order three or higher. The operation of chaotic systems is well-known to be highly sensitive to the values of the parameters of the system, with very small changes of the parameter values producing huge changes in the operation, especially when the parameter values are close to the bifurcation points [16]. Maintaining constant values for all the parameters of a system except the ones that serve as sensing parameters the entire system response will depend on the selected sensing parameter or parameters. Several conditions consisting in regions in the parameter space can be monitored using this technique [17]. This makes chaotic systems most suitable for multi-sensing applications. However, the high sensitivity is also a drawback when the values of some parameters are difficult to control. As an example, the chaotic circuits tend to be very sensitive to the change of the power supply and to the temperature of the circuit; therefore, these parameters have to be well controlled and possibly compensated. However, the compensation of the chaotic circuit is more difficult than for a static (i.e., non-chaotic) nonlinear sensor.

One of the disadvantages of the chaotic sensors and other applied chaotic systems is that they may have a slow response because of a long transitory regime before entering in the "steady-state" nonlinear dynamic regime. This is a limit also known for - and inherited from - periodic oscillators. For performing a measurement with a chaotic sensor after the value of the measured parameter changes, the sensor has to stabilize, that is, it has to go through the transitory dynamic regime and enter its "steady-state" dynamic regime. The duration of the transitory regimes may vary from very short, of a few periods of a linear oscillator and a few quasi-periods of the nonlinear system representing the chaotic sensor, to tens of milliseconds.

For understanding the difference between common parameter-to-frequency converters and chaotic parameter-to-waveform converters, one should 
consider that the former produce (typically) a sinusoidal or a rectangular wave with frequency proportional with the parameter; the latter ones produce a complex waveform that changes when the parameter changes. In the frequency domain, common sinusoidal parameter-to-frequency converters have a singleton at a frequency corresponding to the parameter value. Chaotic parameter-to-waveform sensors have a complex frequency spectrum which changes when the parameter changes. Because the oscillator is nonlinear, the waveform is complex, rich in harmonics even when the oscillation is non-chaotic. The spectral peaks may be almost as high as the maximal peak. In addition, typically there are spectral components at frequencies lower than the frequency of the main component.

\section{SENSOR DESIGN}

Once the appropriate sensing element (capacitance, inductance, or resistance) is chosen for the application, the design of the chaotic sensor starts with the choice of a suitable chaotic circuit. The sensor circuit exemplified in this paper is a variant of the one used in $[7,8]$. It consists of two operational amplifiers in a loop that also contains series capacitors and an RLC circuit $(\mathrm{L} 1, \mathrm{C} 3, \mathrm{R} 6)$ in parallel with another capacitor (C2); the load of one of the amplifiers is the resonant circuit (L2, C4), see Fig. 1. The choice of the operation amplifier is not random: the amplifier internal poles play a major role in chaotic behavior of the circuit, as they contribute to establishing the order of the differential equation governing the circuit. In addition, the poles and zeros due to parasitic circuit elements and the slew rate and the nonlinearities of the amplifiers play an essential role in the nonlinear behavior of the circuit, as is the case with LT1797. The amplifier should support the entire frequency spectrum required by the nonlinear dynamic operation, as dictated by the solutions of the nonlinear differential equations of the circuit. The sensing circuit element is the capacitor $\mathrm{C} 1$. The circuit behaves as an almost sinusoidal oscillator with the values in the scheme for values of $\mathrm{C} 1$ between $0.01 \mathrm{nF}$ and $5 \mathrm{nF}$ and for values larger tha about $100 \mathrm{nF}$. The interval of values of $\mathrm{C} 1$ where the operation of the circuit is chaotic is roughly between $0.3 \mathrm{nF}$ and $0.4 \mathrm{nF}$, for power supply of $\pm 5 \mathrm{~V}$. Because of the low value of R1, the amplifier U1 works with output currents close to the short-circuit condition, which may be an issue with some chips.

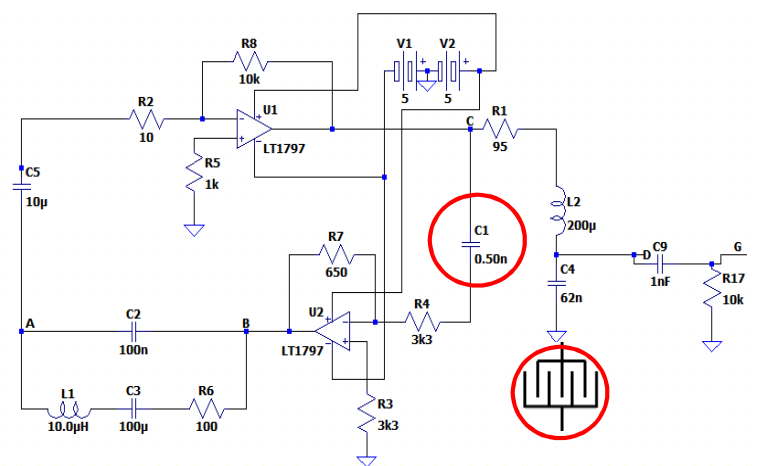

Figure 1. Scheme of the sensing chaotic oscillator. The sensing element in the examples in the text is $\mathrm{C} 1$. In sensor applications, $\mathrm{C} 1$ may be an interdigital capacitor printed on a plastic or textile support.
Several other elements of the circuit can be used as a second sensor, for example C4, L4, or R1. Supplementary notch filters are used to remove undesired components from the spectrum of the signal generated by the chaotic system. The removed undesired components represent spectral peaks related to the main cycle duration of the chaotic attractor; their removal is improves the sensitivity of the sensor and cleans the spectrum of the components close to the 124 or $135 \mathrm{kHz}$ used in RFID technology, when the sensor is to be used as a self-emitting RF tag. The notch filters and the signal amplifier are in Fig. 2.

The sensing element capacitor $\mathrm{C} 1$ can be split between a fixed capacitor of 100 to $250 \mathrm{pf}$ and a sensing capacitor; the latter can be printed on a paper label or on cloth. A basic version of the circuit used in this paper is analyzed in [14] and is demonstrated and pictured in [6].

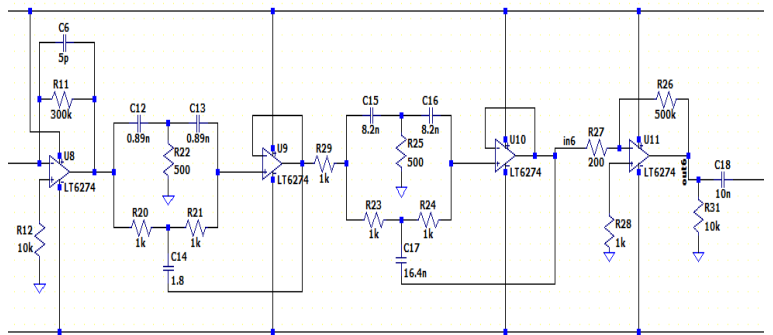

Figure 2. Notch filters are used to remove undesired spectral components in the signal generated by the chaotic oscillator.
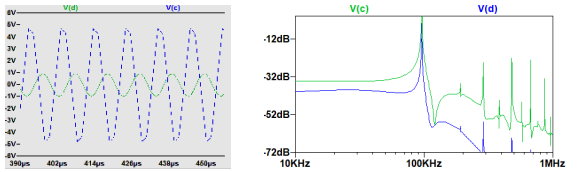

Figure 3. Almost sinusoidal operation for $\mathrm{C} 1=0.1 \mathrm{nF}$.
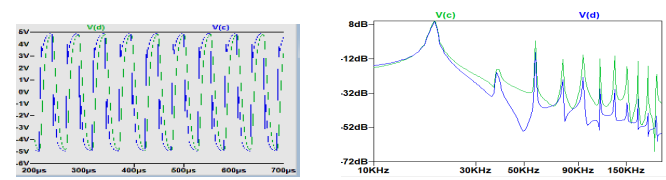

Figure 4. Transition to chaotic regime, at $\mathrm{C} 1=0.15 \mathrm{nF}$.
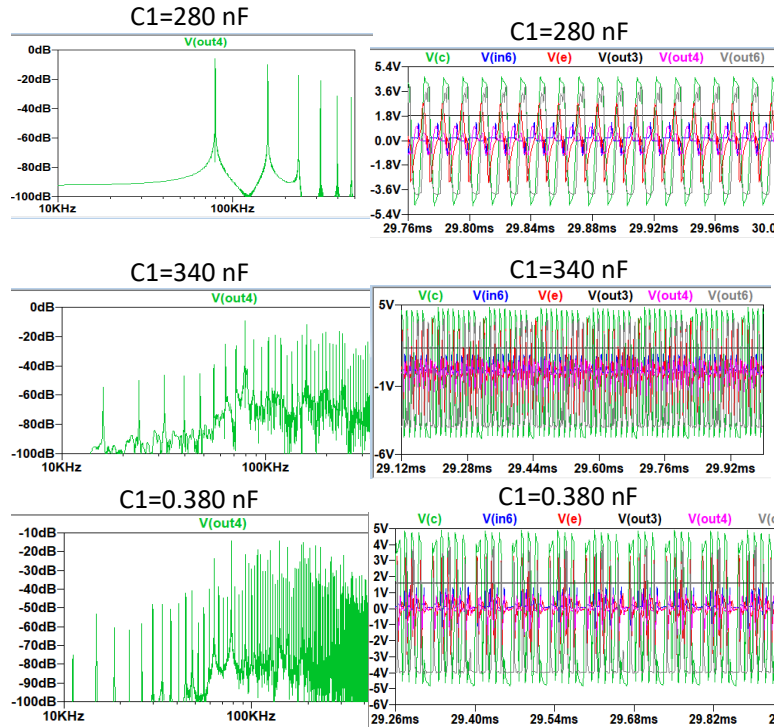

Figure 5. Examples of spectra and waveforms for various $\mathrm{C}$ values. 
When $\mathrm{C} 1$ value is far from the intervals where the operation is chaotic, the circuit operates as an almost sinusoidal oscillator; the periodic operation is exemplified in Fig. 3, for the circuit in Fig. 1 with $\mathrm{C} 1=0.1 \mathrm{nF}$ (Vc is at the output of the amplifier $\mathrm{U} 1 ; \mathrm{Vd}$ is on the capacitor in the output resonant circuit). When the value of $\mathrm{C} 1$ is increased to $1.5 \mathrm{nF}$, the circuit is still periodical, but the generated waveform has a wider spectrum (Fig. 4). As the value of the capacitive sensor $\mathrm{C} 1$ further increases, the system enters in various chaotic regimes, see Fig. 5.

\section{CONVERTING THE CHAOTIC ATTRACTOR AND CHAOTIC OPERATION INTO A SCALAR OUTPUT}

In common sensors, the input (measured) parameter is converting into another scalar parameter, for example measured resistance (as a proxy for temperature, pressure, or stress), into an output voltage. This simple type of mapping is also desired for chaotic sensors, but may be difficult to achieve. One can look at chaotic sensors from several perspectives, as parameter (or vector) to waveform converter, as parameter to frequency spectrum converter, as parameter to attractor converter, or simply as parameter to parameter (scalar) converter, where the second parameter may be, e.g., a voltage.

There are several ways of converting (mapping) the input to a useful output in chaotic sensors. These ways involve time-domain conversion, frequencydomain, or other similar intermediate ways. In the time domain, the rectified waveform average value can be used. In the frequency domain, the amplitude of a specified harmonic may be monitored. More elaboratedly, the chaotic attractor may be characterized by one of its parameters in the phase space, as in [17]. These conversion manners are explained next.

a. Rectified output signal as a proxy of the measured value. This method requires the rectification (half-wave may be more sensitive than full-wave rectification, depending on the specifics of the waveform, i.e., the attractor) and the integration of the rectified waveform for a long enough time. The integration time should be considerably longer than the period of the main spectral component, because the spectrum may be rich in low-frequency components.

b. Spectral component presence (spectrum matching) as an indicator of attaining a specified value of the measured variable. Because the spectrum of the signal is heavily dependent on the measured parameter, the occurrence of a specific spectral component(s) can indicate that the specified valued was reached.

c. Waveform matching for detection of attainment of a specified value. This method is useful when a specific value of the measured variable has to be detected in applications such as threshold level detection in a container, or when checking that a capacitive label has not been tampered in security applications. Waveform matching requires more complex circuitry and a template waveform to compare with. d) Attractor matching. The method was explained in [17].

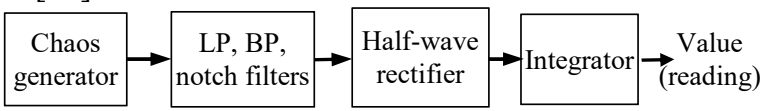

Figure 6. Block diagram for converting the cahotic oscillation output in a scalar output

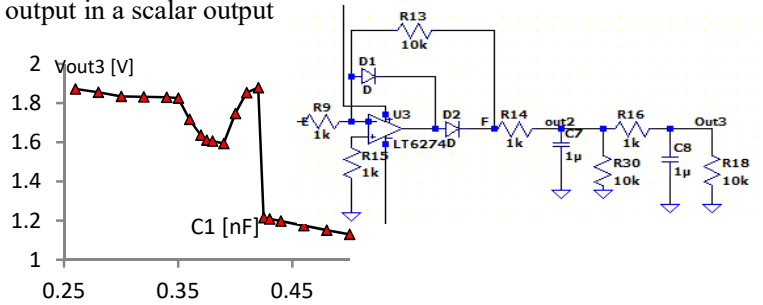

Figure 7. Scalar output obtained by half-wave rectification and integration, see inlay (after $30 \mathrm{~ms}$ ).

The first method is illustrated in Fig. 6, which shows that the chaotic circuit can detect minute $(<5 \mathrm{pF}$ at $425 \mathrm{pF}$ ) changes in the circuit; that makes them suitable for sensitive sensing. The single-polarity rectifier-integrator method has its own drawbacks: the integration results are stable after long integration time (about $30 \mathrm{~ms}$ in this case).

\section{APPLICATIONS: TAGS}

The design refers to sensors that are suitable to develop passive or active tag-sensors. A self-signaling, level threshold sensor is a direct application of the described circuit. The application is based on sensing the level through the change in capacitance $(\mathrm{C} 1)$, where $\mathrm{C} 1$ is an interdigital capacitor on a label positioned at the desired level on a vessel (assuming that the vessel wall is a dielectric with low permittivity and that the liquid or powder in the vessel has high permittivity). The configuration of $\mathrm{C} 1$ should be trimmed such that before the fluid attains the desired level, the value of $\mathrm{C} 1$ is less than $420 \mathrm{pF}$, increasing above $425 \mathrm{pF}$ when the level is attained (see Fig. 7). When the sensor is power wired (connected in a system by wires), a rectifier and integrator are used and the voltage jump at the output of the integrator is detected and sent as output.

With small changes, the sensor can operate as a passive or active emitting tag. The operation of the chaotic circuit may be powered by local batteries or by an RF-reader. When used in passive mode, a specific requirement is that the chaotic regime has to be attained during the small lapse of time when the RF reader operates - a few tens of ms (typically charge time is 25 to $100 \mathrm{~ms}$ ); the requirement is satisfied, as the chaotic operation starts after less than $20 \mathrm{~ms}$ (worst case). In this type of application, the output of U11 in Fig. 2 is connected to an antenna $(135 \mathrm{kHz})$.
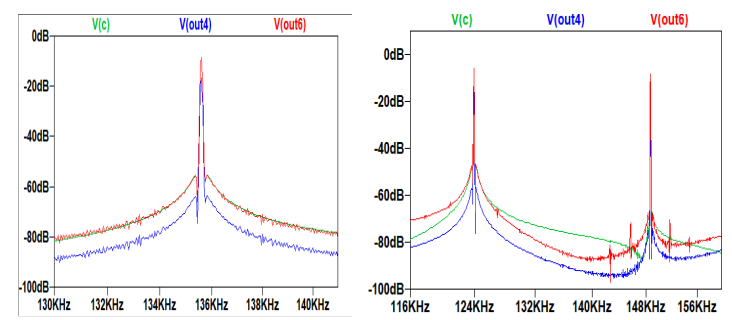

Figure 8. Tuned at $\mathrm{C} 1=0.430 \mathrm{nF}$ (left) and detuning at $420 \mathrm{nF}$ (right). 
Alternatively, a piezoelectric generator $(135 \mathrm{kHz})$ may be used for signaling through ultrasound at close distance that the level was reached. The amplitude of the spectral components around $135 \mathrm{kHz}$ change more than $60 \mathrm{~dB}$ around $\mathrm{Cl}=0.43 \mathrm{nF}$ (Fig. 8); that change can be detected at short distances. A two-stage second order BPF was used to add an extra $30 \mathrm{~dB}$.

\section{DISCUSSION AND CONCLUSIONS}

While a single circuit was discussed in this study, there are other suitable circuits that can fit the presented applications, for example the circuits described in [17], [18].

The presented results and design methods are relatively easy to employ in a variety of applications, ranging from industrial to medical. When designing with chaotic sensors one has, however, to consider their disadvantages, including the need of a stable and precise power supply (in most cases), high temperature sensitivity, and indirect determination of the measured value and more complex circuits to extract the measured value. Even for simpler applications such as level threshold detection, the disadvantages persist.

Further research is needed to develop sensors with the antenna or a capacitor loading the antenna performing as the sensing element. Such chaotic circuit whose operation is strongly dependent on the antenna or on the value of a capacitor loading the antenna could be more sensitive and at the same time lower cost. Application of the proposed solution to tampering detection $[19,20]$ and other uses described in [21] seems also promising.

\section{ACKNOWLEDGMENT}

Dr. V. Cojocaru collaborated during former studies on the topic. An early circuit in Fig. 1 was made by both of us.

\section{REFERENCES}

[1] F. Krummenacher, "A High-Resolution Capacitance-toFrequency Converter," in IEEE Journal of Solid-State Circuits, vol. 20, no. 3, pp. 666-670, June 1985, doi: 10.1109/JSSC.1985.1052366.

[2] Chatzandroulis, S., Tsoukalas, D. Capacitance to Frequency Converter Suitable for Sensor Applications Using Telemetry. Analog Integrated Circuits and Signal Processing 27, 31-38 (2001). https://doi.org/10.1023/A:1011290419804.

[3] Matko, V., \& Milanović, M. (2014). High resolution switching mode inductance-to-frequency converter with temperature compensation. Sensors (Basel, Switzerland), 14(10), 19242-19259. https://doi.org/10.3390/s141019242.

[4] H.N.L. Teodorescu, Patent application RO9500903A, 199505-12, Publication RO115316B1·1999-12-30, Non-linearoscillator proximity transducer. https://worldwide.espacenet.com/ patent/search/family/064357730/publication/RO115316B1?q $=\mathrm{pn} \% 20 \mathrm{any} \% 20 \% 22 \mathrm{RO} 115316 \mathrm{~B} 1 \% 22$ )

[5] Teodorescu, H.N.: Method for measuring at least one parameter and device for carrying out this method. European Patent Application \#988-10802.3, Aug. 1998 / 23.02.2000. http://ep.espacenet.com/ .

[6] H.N.L. Teodorescu and V.P. Cojocaru, "Biomimetic chaotic sensors for water salinity measurements and conductive titrimetry," 2012 Third IEEE International Conference on Emerging Security Technologies (EST), Lisbon, 2012, pp. 182-185, doi: 10.1109/EST.2012.42.
[7] H.N. Teodorescu, Kandel A., Grigoras F., Mlynek D. (2002a), "Measuring with chaos: Sensorial systems and A/tganglions." Proc. Romanian Academy, Series A, 3, (1-2), 2002, pp. 55-62. http://www.acad.ro/sectii2002/proceedings/proc_pag2002_n0 1.htm (Accessed 15 July 2020).

[8] F. Yejia, L. Wenshi, X. Peng and C. Li, "Chaos based blood glucose noninvasive measurement: concept, principle and case study," 2016 Sixth International Conference on Instrumentation \& Measurement, Computer, Communication and Control (IMCCC), Harbin, 2016, pp. 340-343, doi: 10.1109/IMCCC.2016.196.

[9] H.N. Teodorescu et al., "An artificial retina based on chaotic neuronal ganglions, Proc. Int. Invitational Workshop on Intelligent Interface Devices. Supported by the Japan Society for the Promotion Science under Grant-In-Aid for Research for the Future Program. Mar 13-14, pp. 39-43, 2002 Kitakyushu, Japan.

[10] D.N. Butusov, T.I. Karimov, O.S. Druzhina, A.I. Karimov Development of inductive sensor based on random oscillator. Вестник Брянского государственного технического университета № 12 (85) 2019, pp. 82-93.

[11] Karimov, T., Nepomuceno, E. G., Druzhina, O., Karimov, A., \& Butusov, D. (2019). Chaotic Oscillators as Inductive Sensors: Theory and Practice. Sensors (Basel, Switzerland), 19(19), 4314. https://doi.org/10.3390/s19194314.

[12] W. Korneta, G. Iacyel, P. Rodrigo and M. Zabovsky, "Nonlinear sensor driven by chaotic dynamics," ANNA '18; Advances in Neural Networks and Applications 2018, St. Konstantin and Elena Resort, Bulgaria, 2018, pp. 1-4. ANNA '18; Advances in Neural Networks and Applications 2018.

[13] T. I. Karimov, M. V. Antonovich, A. S. Mashanin, A. S. Yastrebkov and A. I. Slizh, "Synthesis of Chaotic Circuits with Inductive Elements Based on 3rd Order Differential Equations," 2019 IEEE Conference of Russian Young Researchers in Electrical and Electronic Engineering (EIConRus), Saint Petersburg and Moscow, Russia, 2019, pp. 98-101, doi: 10.1109/EIConRus.2019.8657066.

[14] H.N.L. Teodorescu, V.P Cojocaru, Complex signal generators based on capacitors and on piezoelectric loads, 2011, In: Chaos Theory: Modeling, Simulation and Applications, Pages 423-430, World Scientific Publishing Co Pte Ltd., ISBN \#9789814350341, DOI: 10.1142/9789814350341_0049.

[15] D. Poljak, M. Cvetković, Chapter 3, Incident Electromagnetic Field Dosimetry, 3.2.2 Fields in the Vicinity of RFID Loop Antennas. In: Human Interaction with Electromagnetic Fields, Computational Models in Dosimetry. 2019, Pages 53-89, 2019, Academic Press, ISBN 978-0-12-816443-3.

[16] H.N.L. Teodorescu, Modeling natural sensitivity: Alife sensitive, selective sensors. International Journal of Biomedical Soft Computing and Human Sciences: the official journal of the Biomedical Fuzzy Systems Association, 2000, Volume 6, Issue 1, Pages 29-34 Biomedical Fuzzy Systems.

[17] H.N.L. Teodorescu, "Sensors based on nonlinear dynamic systems - A survey," 2017 International Conference on Applied Electronics (AE), Pilsen, 2017, pp. 1-10, doi: 10.23919/AE.2017.8053572

[18] M. Stork, J. Hrusak, D. Mayer, "A new chaotic system based on state space energy feedback". IEEE Int. Conf. Applied Electronics (AE), Pilsen, Czech R., 2015, pp. 229-232.

[19] H. Eren, L.D Sandor, "Fringe-effect capacitive proximity sensors for tamper proof enclosures." 2005 Sensors for Industry Conf., 8-10 Feb. 2005, Print ISBN: 1-4244-0298-0, DOI: 10.1109/SICON.2005.257863, Houston, TX, USA.

[20] E. Ferres, V. Immler, A. Utz, A. Stanitzki, R. Lerch, R. Kokozinski, Capacitive multi-channel security sensor IC for tamper-resistant enclosures. 2018 IEEE SENSORS, 28-31 Oct. 2018, New Delhi, India, DOI: 10.1109/ICSENS.2018.8589716.

[21] C. Occhiuzzi, S. Caizzone and G. Marrocco, "Passive UHF RFID antennas for sensing applications: Principles, methods, and classifcations," in IEEE Antennas and Propagation Magazine, vol. 55, no. 6, pp. 14-34, Dec. 2013, doi: 10.1109/MAP.2013.6781700 